\title{
Modelagem Computacional no Estudo de Deformações em Pavimentos Flexíveis
}

\section{Computational Modeling in the Study of Deformation in Flexible Pavements}

Marcelo Molin ${ }^{1}$, Leandro Blass², Anderson L. J. Bihain ${ }^{3}$, Lucas Dotto Bueno4, Jaelson Budny ${ }^{5}$, Maicon Vinicius Altnetter ${ }^{6}$, Ademilton Luiz Rodrigues de Souza $^{7}$, João A. G. Kazmirski ${ }^{8}$, Everson Jonatha Gomes da Silva ${ }^{9}$.

\section{RESUMO}

Devido a matriz dos transportes brasileira ser constituída em grande parte por deslocamentos rodoviário, torna-se importante conhecer os principais fatores que influenciam a ruína de uma rodovia, por conta disto o presente trabalho visa estudar o comportamento de um pavimento exposto a uma variação sazonal de temperatura, assim permitindo a análise das respostas estruturais quando submetido aos carregamentos dos eixos rodoviários. Para realizar esta análise, levaram-se em conta três tipos de revestimentos de espessuras diferentes $(5,10$ e $15 \mathrm{~cm})$ considerando que o módulo de resiliência destas camadas varia com a temperatura. Os dados experimentais de temperatura são referentes a média de dez dias de forte insolação do mês de fevereiro das 13:00h horas às 18:20h variando de 20 em 20 minutos. Através do software AEMC/SisPav, que utiliza os módulos calculados para cada seção do revestimento e os módulos referentes as demais camadas do pavimento, serão calculadas as deformações, tensões e deslocamentos para estimar sua vida útil através de modelos de desempenho que simulam a degradação da estrutura através dos mecanismos de ruptura por fadiga e deformação permanente.

Palavras-chave: Pavimento flexível, Variação da temperatura, Módulo de resiliência, Respostas Estruturais.

Due to Brazilian transport infrastructure logistics chain to be mainly supported by road transport it is important to know the main factors that lead roads to ruin, because of this, the present work aims to study the behavior of a pavement exposed to a seasonal variation of temperature, which will allow the structural responses analysis when is subjected to the loadings of the road transport. To perform this analysis, three types of coatings of different thicknesses $(5,10$ and $15 \mathrm{~cm})$ were taken into account, was considered that the resilience modulus of these layers varies with the temperature. Experimental temperature data refers to the average of ten days of strong insolation in the month of February from 13:00 a.m. to 18:20 a.m. ranging from 20 in 20 minutes. Through the AEMC / SisPav software, that uses the calculated modules for each coating section, and the modules referring to the other layers of the pavement, it will be calculated the deformations, stresses, and displacements to estimate their useful life through performance models that simulate degradation of the structure through the mechanisms of fatigue rupture, and permanent deformation.

Keywords: Flexible Pavement, Temperature Variation, Resilience Module, Structural Responses.

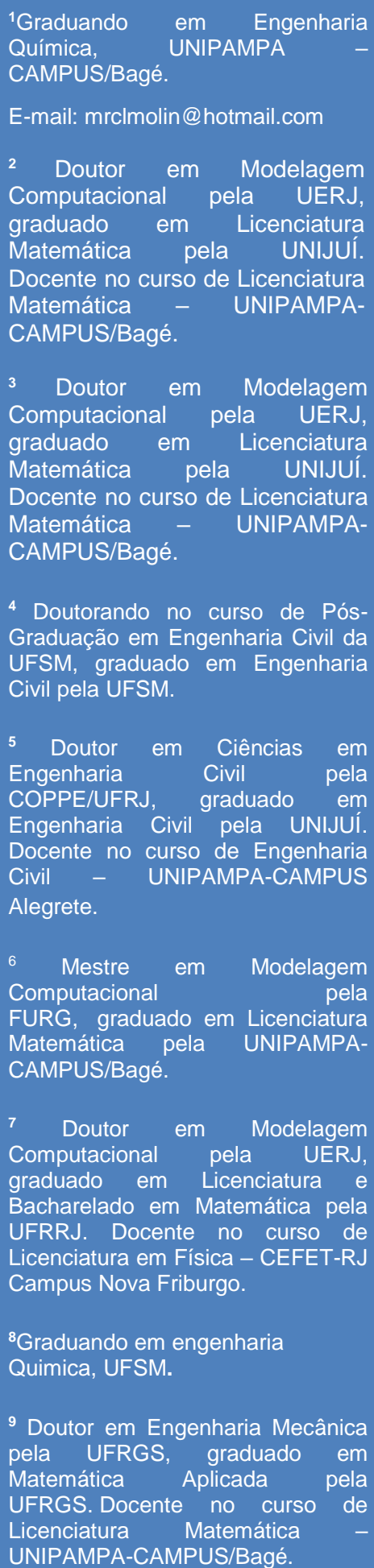

4 Doutorando no curso de PósGraduação em Engenharia Civil da UFSM, graduado em Engenharia Civil pela UFSM. 


\section{INTRODUÇÁO}

Pavimentos são estruturas que visam tornar mais prático o rolamento ou tráfego de mercadorias e pessoas, sua vida útil depende de inúmeros fatores físicos e mecânicos das camadas que o constitui. Por seu revestimento possuir em sua composição derivados do petróleo (ligante asfáltico) pode-se considera-lo uma estrutura termo sensível (BALBO, 2007).

A distribuição espacial da logística de transportes no território brasileiro revela uma predominância do modal rodoviário. Segundo pesquisa da Confederação Nacional dos Transportes (CNT) do ano de 2017, do total de rodovias analisadas 61,8\% apresentam algum tipo de irregularidade. Devido a estes problemas oriundos da importância do transporte rodoviário e da qualidade de sua estrutura, se torna importante conhecer o que causam estas avarias e como pode-se evita-las ou eliminalas.

Os dois principais defeitos presente em rodovias brasileiras, são deformações por fadiga e deformação permanente (afundamento de trilha de roda), ambas deformações afetam a capacidade de rolagem de uma rodovia a tornando menos segura (MEDINA e MOTTA, 2015). Para estima-los utiliza-se modelos de desempenho que são equações matemáticas que simulam um dano a um pavimento buscando prever o desempenho do mesmo.

Fadiga é um processo de deterioração a que está sujeito um material ao ser submetido a tensões e deformações repetidas, resultando em trincas após certas repetições (PINTO,1991). Para o (DNIT, 2003) a deformação permanente é caracterizada pelos afundamentos ao longo da trilha da roda, sendo notável uma depressão na superfície do pavimento, sendo causadas por deformações de compressão.

Resiliência é a máxima energia que um material pode receber sem sofrer deformação, já o módulo de resiliência é a deformação por unidades de volume. Quanto menor for o módulo de resiliência menor será a rigidez do material (HIBBELER, 2010). Por ser uma propriedade que varia com a temperatura é um parâmetro importante para analisar-se a influência da variação da mesma nos pavimentos asfálticos.

Segundo (W. ALKASAWNEH et al., 2007) a variação de temperatura ao longo do perfil do pavimento é afetada principalmente pela alteração da temperatura ao longo da superfície do mesmo, que varia continuamente durante os meses. Assumir um único módulo de resiliência para o pavimento pode superestimar ou subestimar as 
propriedades de rigidez do mesmo dependendo das condições climáticas prevalecentes durante um ano.

Através de dados experimentais de temperatura (BLASS, 2011) coletados para um pavimento experimental localizado na cidade de ljui/RS com o auxílio de termopares distribuídos pelo mesmo, foi possível, estimar as temperaturas para determinadas profundidades do pavimento através da modelagem computacional da transferência de calor.

Para conhecer a deformação horizontal de tração $\left(\mathcal{E}_{t}\right)$ na fibra inferior do revestimento, a deformação vertical de compressão no topo do subleito $\left(\varepsilon_{c}\right)$ e a deflexão na fibra superior do revestimento $\left(U_{z}\right)$ referente à atuação de um eixo padrão, utilizou-se a ferramenta $\mathrm{AEMC/SisPav}$, que realiza seus cálculos através da teoria elástica de multicamadas de Burmister. Consideraram-se, na rotina de cálculos do AEMC, módulos de resiliência variáveis de acordo com as temperaturas obtidas pela modelagem.

Tais parâmetros podem ser usados para estimar a vida útil de um pavimento, por exemplo, calculando-se o número $\mathrm{N}_{\mathrm{f}}$, que seriam os valores de solicitações que levam o pavimento a ruína por fadiga ou $N_{\text {atr }}$ deformação permanente afundamento por trilhas de roda. Optou-se por calcular o número $\mathrm{N}_{\mathrm{f}}$ e $\mathrm{N}_{\text {atr }}$ para um eixo padrão, eixo simples de rodas duplas com carga de 8,2 toneladas e pressão dos pneus de 0,56 Mpa, pois este é o eixo utilizado nos métodos vigentes de dimensionamento de pavimentos asfálticos.

O presente trabalho tem por objetivo estimar $\mathcal{E}_{\mathrm{t}}, \mathcal{E}_{\mathrm{c}}, \mathrm{U}_{z}$ além do $N_{\text {atr }}$ (valor de solicitações que levariam o pavimento a ruína por afundamento de trilha de roda) e do $\mathrm{N}_{f}$ (valor de solicitações que levariam o pavimento a ruína por fadiga) para três pavimentos diferentes quanto ao seu revestimento sendo 5,10 e $15 \mathrm{~cm}$ de medidas para este, e $25 \mathrm{~cm}$ de base para todos e também compará-los com o modelo proposto de cálculo pelo DNIT que utiliza uma temperatura padrão de $25^{\circ} \mathrm{C}$.

\section{MATERIAIS E METODOS}

Para o cálculo do módulo de resiliência foi utilizada a seguinte equação proposta por (ULLIDTZ, 1987) em função da temperatura.

$$
M_{r}=15000-7900 \log (\mathrm{T})
$$


onde, $\mathrm{Mr}$ é o módulo de resiliência em Mpa e T é a temperatura em ${ }^{\circ} \mathrm{C}$.

As temperaturas foram retiradas de (BLASS, 2011) para o mês de fevereiro (das 13:00h até as 18:20h), a partir de seis sensores instalados em um pavimento flexível experimental existente no Laboratório da Engenharia Cívil da UNIJUí, Universidade Regional do Noroeste do Estado do Rio Grande do Sul.

Estes sensores localizam-se na superfície do revestimento, a 3, 6, 9,12 e $15 \mathrm{~cm}$ de profundidade, e através dos dados coletados de 20 em 20 minutos fez-se uma modelagem, utilizando o método de diferenças finitas, a fim de se conhecer a temperatura do revestimento de $1 \mathrm{em} 1 \mathrm{~cm}$ e posteriormente o módulo de resiliência referente a cada centímetro do mesmo.

Também se calculou o modulo de resiliência para a temperatura padrão de $25^{\circ} \mathrm{C}$, valor que norteia os principais testes e avaliações de pavimentos recomendados pelo departamento nacional de infraestrutura e transportes (DNIT), (DNIT, 2006).

Como ferramenta no auxílio dos cálculos utilizou-se da mecânica de pavimentos através da ferramenta de Análise Elástica de Múltiplas Camadas (AEMC/SisPav), que é um sistema computacional formulado em $\mathrm{C}++$, desenvolvido por (FRANCO, 2007). O software é específico para o cálculo de tensões, deformações e deslocamentos, com rotinas para entrada de dados e apresentação de resultados.

Para este trabalho buscou-se os valores de $\mathcal{E}$ t, $\mathcal{E} \mathrm{c}$ e $U z$ os dois primeiros para serem usados nos modelos de desempenho (modelo de trincamento por fadiga e afundamento de trilha de roda) e o último foi utilizado apenas como embasamento dos resultados.

Os pontos de análise para se obter tais parâmetros são os pontos em que o eixo adotado (eixo padrão) atua, neste caso tais pontos baseiam-se em (RIBAS, 2014), no centro das rodas, na borda interior e no centro da roda externa. Para este tipo de eixo trabalha-se na direção transversal do eixo $(\mathrm{X})$ mantendo a direção longitudinal $(\mathrm{Y})$ nula, conforme Figura 1. 


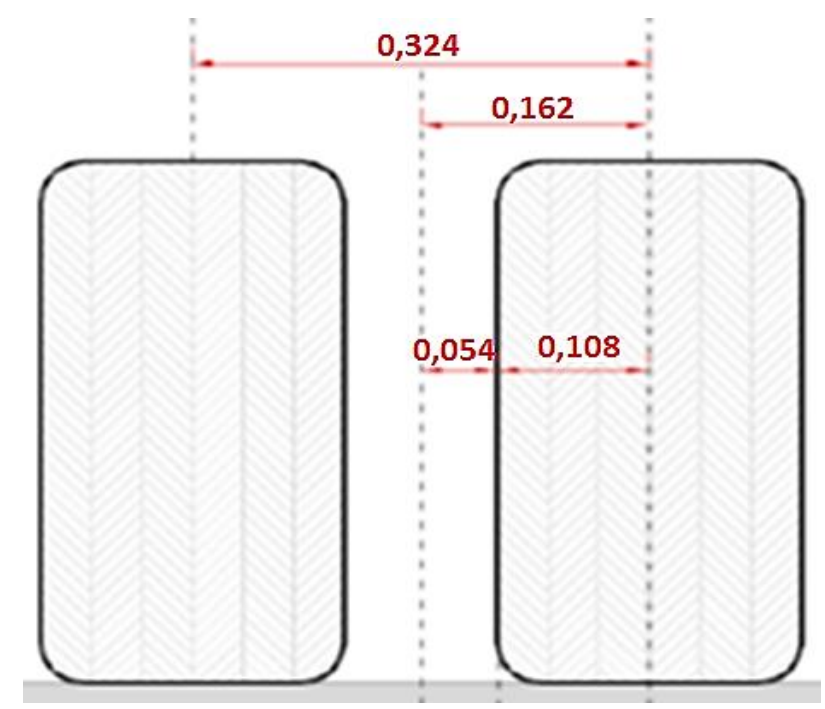

Figura 1. Pontos de análise eixo padrão. Fonte: (RIBAS ,2014).

Para as camadas do pavimento, considerando o eixo Z, serão então coletados dados: na fibra superior do revestimento $U_{z}$, na fibra inferior do revestimento para $\mathcal{E}_{\mathrm{t}}$, e na fibra superior do subleito para $\mathcal{E}_{\mathrm{c}}$, conforme a Figura 2 (FRANCO, 2007).

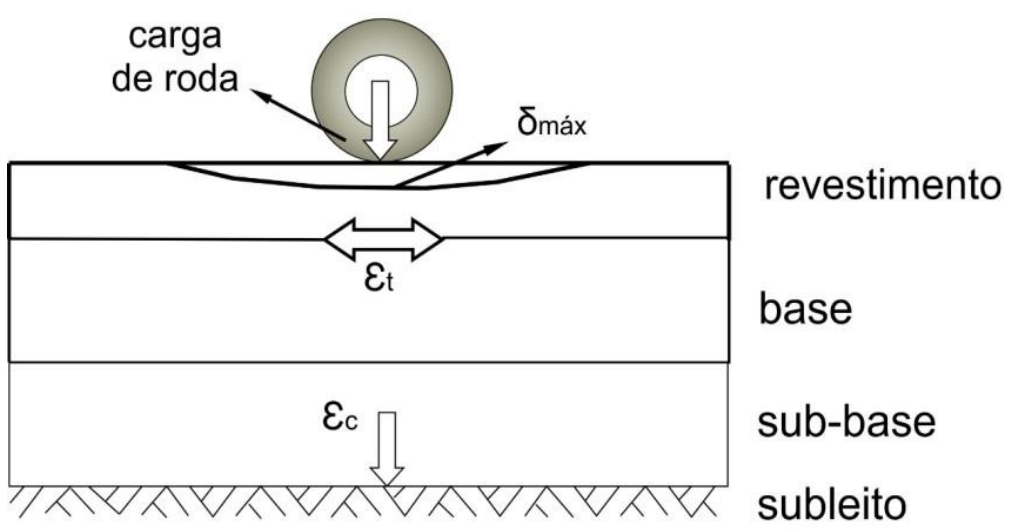

Figura 2. Locais de análise em relação à profundidade.

Fonte: (FRANCO, 2007).

O modelo proposto pela Federal Highway Administration (FHWA) foi obtido através de dados experimentais e relaciona a deformação de tração $\mathcal{E}_{t}$, no qual podemos $0 \mathrm{~N}$ (ROSSATO, 2015) da seguinte forma:

$$
N_{f}=1,092 \cdot 10^{-6}\left(\frac{1}{\varepsilon_{t}}\right)^{3,512}
$$


onde, $\mathrm{N}_{\mathrm{f}}$ é o número das solicitações que levariam o pavimento a ruína por fadiga. Já o calculo do Natr para deformação permanente usamos da informação deformação por compressão $\mathcal{E}_{\mathrm{c}}$, pelo modelo da Asphalt Institute Design Method (BUENO, 2014):

$$
N_{\text {atr }}=1,365 \cdot 10^{-9} \cdot \varepsilon_{c}^{-4,477}
$$

De modo que, Natr é o número de solicitações que levariam o pavimento a ruína por afundamento de trilho de roda.

\section{RESULTADOS}

Com os valores obtidos no software foi possível traçar gráficos, com as respostas estruturais para as diferentes espessuras de revestimentos durante atuação da carga eixo padrão e comparar a influência da temperatura e espessura do revestimento, bem como a forma que o pavimento responderia segundo as especificações do DNIT (todo o pavimento a $\left.25^{\circ} \mathrm{C}\right)$. A Figura 3 representa o deslocamento que acontece quando 0 pavimento é submetido à carga.

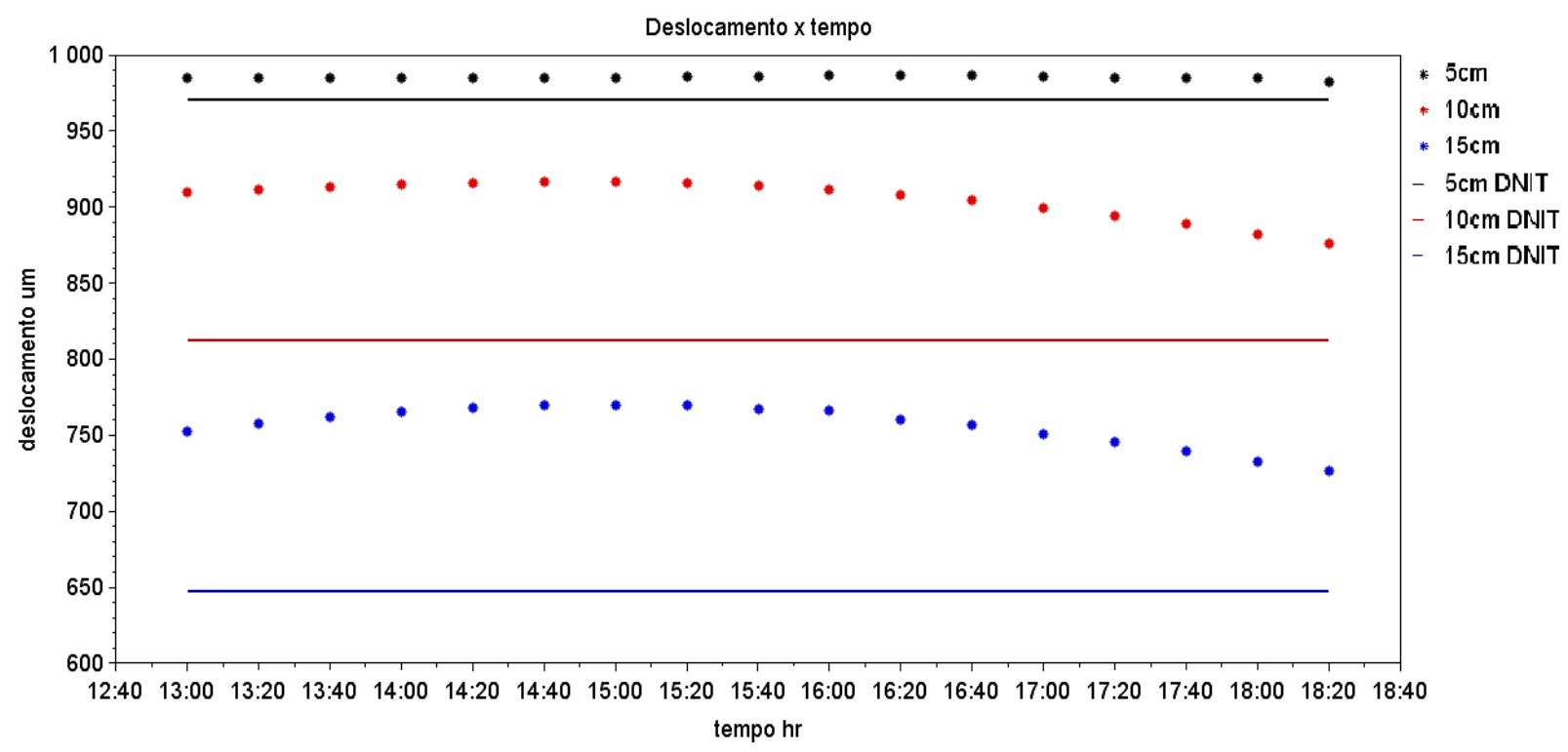

Figura 3. Deflexão $(\mu \mathrm{m}) \times$ tempo.

A Figura 4 representa a deformação de tração $\varepsilon_{t}$, quando submetido à carga do eixo padrão, ela atua na fibra inferior do revestimento. 


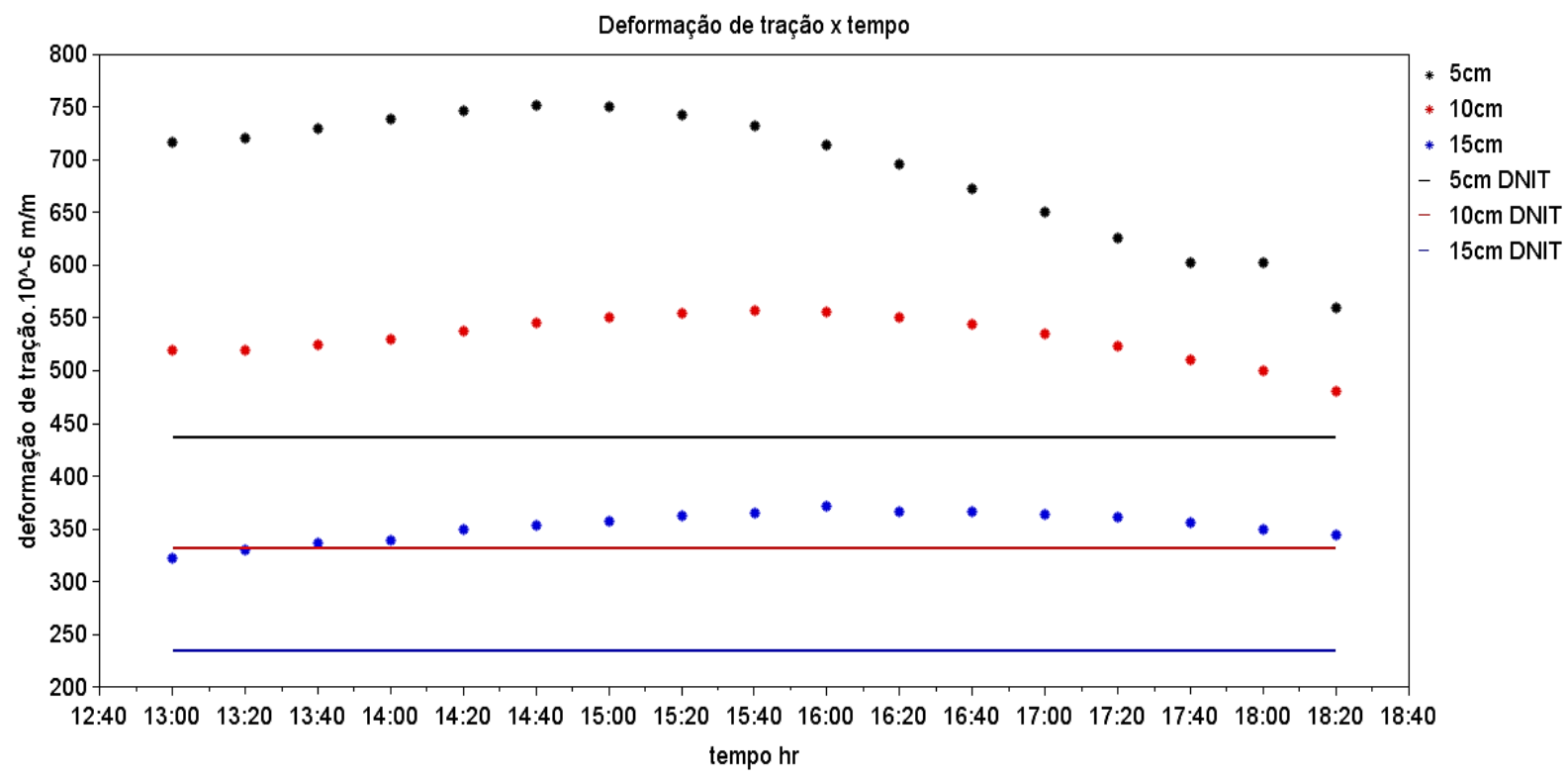

Figura 4. Deformação de tração microstrain $(\mu \mathrm{s}) \times$ tempo.

A Figura 5 representa a deformação de compressão $\mathcal{E}_{c}$, quando submetido a carga do eixo padrão, ela atua na fibra superior do subleito.

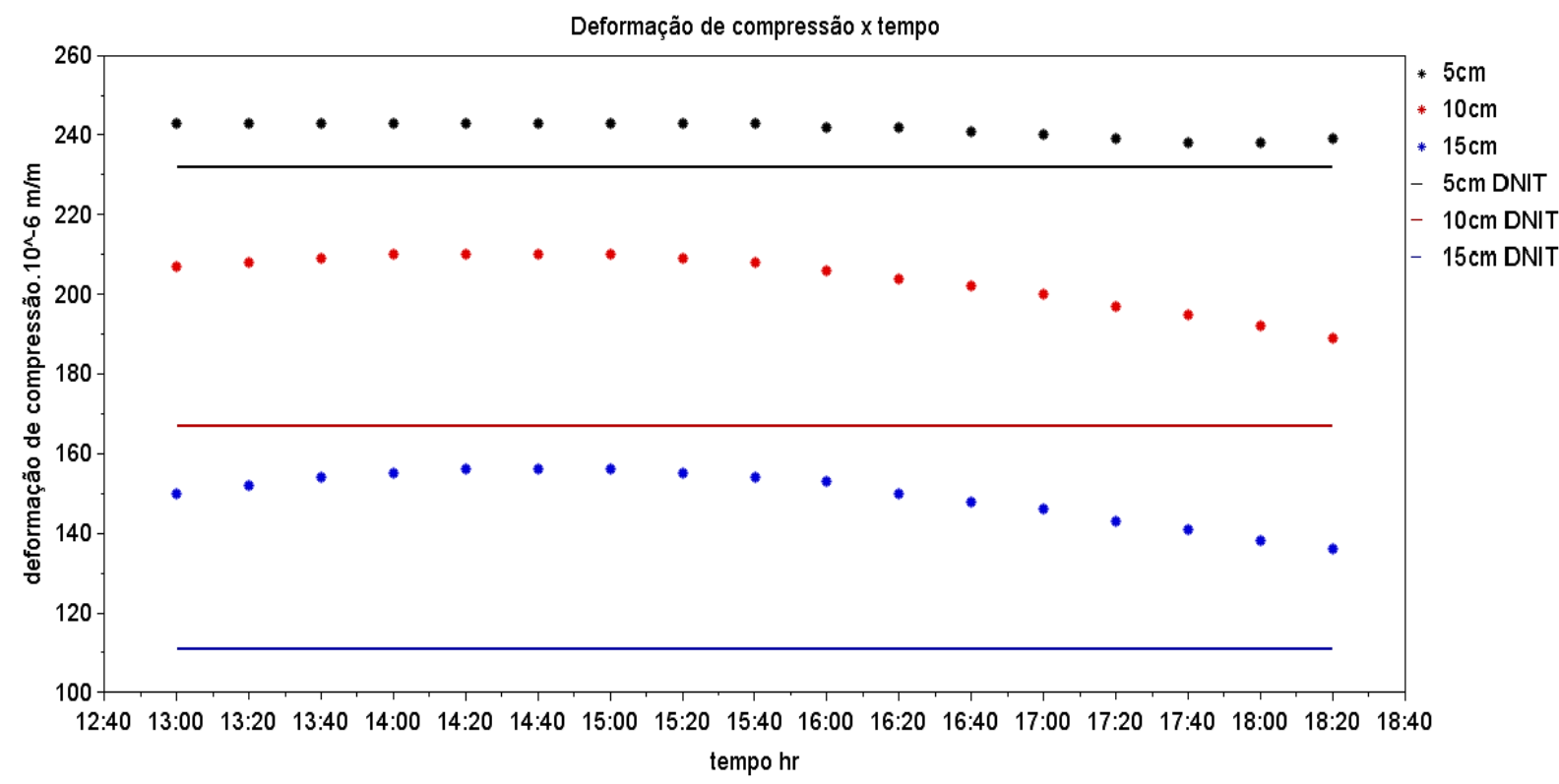

Figura 5. Deformação de compressão microstrain $(\mu s) x$ tempo.

Quanto ao $\mathrm{N}_{f}$ e ao $\mathrm{N}_{\text {atr }}$ calculados para tais períodos, localizou-se o menor valor para tais números de modo a obter-se o horário critico em que o pavimento estaria mais 
vulnerável as deformações referentes a cada número de solicitações, para os horários em análise.

Tabela 1. $\mathrm{N} f$ e $\mathrm{Natr}$ (valores de solicitações).

\begin{tabular}{|c|c|c|c|c|c|}
\hline $\begin{array}{l}\text { Profundidade } \\
\text { do revestimento }\end{array}$ & Abordagem & Horário & $\mathbf{N}_{\mathrm{f}}$ & Horário & $\mathrm{N}_{\text {atr }}$ \\
\hline \multirow[t]{2}{*}{$5 \mathrm{~cm}$} & T variável & $14: 40 \mathrm{~h}$ & $1,021 \times 10^{5}$ & $13: 00 \mathrm{~h}$ & $2,074 \times 10^{7}$ \\
\hline & DNIT - T $=25^{\circ} \mathrm{C}$ & & $6,868 \times 10^{5}$ & & $2,552 \times 10^{7}$ \\
\hline \multirow[t]{2}{*}{$10 \mathrm{~cm}$} & T variável & $15: 40 \mathrm{~h}$ & $2,929 \times 10^{6}$ & $14: 00 \mathrm{~h}$ & $3,986 \times 10^{7}$ \\
\hline & DNIT $-\mathrm{T}=25^{\circ} \mathrm{C}$ & & $1,803 \times 10^{6}$ & & $1,112 \times 10^{8}$ \\
\hline \multirow[t]{2}{*}{$15 \mathrm{~cm}$} & T variável & $16: 00 \mathrm{~h}$ & $1,221 \times 10^{6}$ & $14: 20 \mathrm{~h}$ & $1,508 \times 10^{8}$ \\
\hline & DNIT $-\mathrm{T}=25^{\circ} \mathrm{C}$ & & $6,068 \times 10^{6}$ & & $6,922 \times 10^{8}$ \\
\hline
\end{tabular}

\section{DISCUSSÃO}

No gráfico da Figura 3 pode-se analisar a deflexão, nota-se que dentre os três tipos de revestimentos em questão o mais espesso sofre uma menor deflexão, percebe-se também que o modelo do DNIT subestima tal situação. Os maiores deslocamentos foram medidos no entorno das 15 horas, sendo que o pavimento de revestimento de $5 \mathrm{~cm}$ apresentou deformações quase que lineares.

Quanto à deformação de tração representada pela Figura 4, percebe-se que um revestimento de maior espessura responde melhor a tal deformação, sabendo que maiores deformações representam esforços mais severos repassados ao pavimento pelo eixo comercial, através da superfície do pavimento pode-se perceber que assumir que todo o pavimento se encontra a uma temperatura de $25^{\circ} \mathrm{C}$ novamente não representa os casos mais críticos. Considerando o revestimento de $15 \mathrm{~cm}$ a maior deformação aparece às 15 horas já para o revestimento de $10 \mathrm{e} 5 \mathrm{~cm}$ fica aproximadamente pelas 16 horas.

Quanto à deformação de compressão novamente percebemos valores maiores sobre o pavimento mais delgado de modo que os esforços passados ao mesmo pelo eixo comercial são mais intensos. Em relação a tal deformação apresentou-se picos entre as 14 e as 15 horas e novamente o pavimento com revestimento de $5 \mathrm{~cm}$ mostrou deter um comportamento muito próximo do linear.

A Tabela 1 traz as informações das respostas estruturais: se for considerado a temperatura constante com proposto pelo DNIT ele prevê uma durabilidade muito superior aos encontrados nas respostas estruturais considerando a variação sazonal de 
temperatura. Isso pode ser uma das maiores causas da pavimentação não atingir a durabilidade proposta nos projetos.

\section{CONSIDERACOOES FINAIS}

Conhecendo as condições climáticas a que um pavimento está submetido, pode-se estimar os esforços e respostas que o mesmo sofrerá ao lhe ser imposto uma força, visto que sua rigidez está intimamente atrelada a temperatura a que se encontra tal estrutura, devido as características térmicas da mesma. Logo o módulo de resiliência pode ser expresso como função da temperatura.

Percebe-se uma relação entre a espessura do revestimento e as respostas a ações de uma carga, sendo que para revestimentos mais robustos as forças decorrentes de tais ações em sua superfície se tornam mais amenas, dessa forma prolongando a vida útil do pavimento de acordo com os modelos de desempenho anteriormente listados.

A partir deste estudo pode-se notar que existem influências nos esforços e na previsão da vida útil do pavimento, de acordo com os mecanismos de ruptura avaliados, considerando a variação da temperatura na camada do revestimento asfáltico. Demonstrando assim que existe necessidade de serem avaliados estes parâmetros corretamente em cada ponto em que a rodovia será solicitada pelo carregamento.

\section{REFERÉNCIAS}

ALKASAWNEH, Wael, PAN, ERNIAN, HAN, FENG, ZHU, RONGHUA and GREEN, ROGER (2007) Effect of temperature variation on pavement responses using 3D multilayered elastic analysis, International Journal of Pavement Engineering, 8:3, 203 212.

BUENO, L. D. Determinação do potencial destrutivo do eixo duplo direcional no dimensionamento de pavimentos flexíveis, Santa Maria, 2014.

BALBO, J. T. Pavimentação Asfáltica: materiais, projeto e restauração. São Paulo: Oficina de Textos, 2007.558p.

\section{BLASS, L. Modelagem Matemática de Respostas Estruturais em Pavimentos} Flexíveis Considerando a Variação Sazonal de Temperatura. ljuí 2011.

FRANCO, F.A.C.P. Método de dimensionamento mecanístico-empírico de pavimentos asfálticos - SISPAV. Tese de Doutorado.COPPE/ UFRJ - Universidade Federal do Rio de Janeiro, Rio de Janeiro, 2007. 
HIBBELER, Russell Charles. Resistência dos materiais 7. ed. - São Paulo : Pearson Prentice Hall, 2010.

Ribas, J. Parâmetros elásticos típicos de materiais de pavimentos flexíveis do Rio Grande do Sul e sua aplicação em uma análise de custo/benefício. Santa Maria, 2014.

ROSSATO, F. P. Avaliação do fenômeno de fadiga e das propriedades elásticas de misturas asfálticas com diferentes ligantes em variadas temperaturas. Santa Maria, 2015.

MEDINA, J. MOTTA, L. M. G. Mecânica dos Pavimentos. 2 ed. Rio de janeiro: UFRJ, Interciência 2005.

Pesquisa CNT de rodovias 2017: relatório gerencial. - Brasília: CNT SEST SENAT, 2017.

PINTO, S. Estudo do Comportamento a Fadiga de Misturas. Betuminosas e Aplicação na Avaliação Estrutural de Pavimentos. Rio de Janeiro, 1991.

SITE OFICIAL DO DNIT, disponível em <http://www.dnit.gov.br/>. Acesso em 30 de maio de 2018. 